\title{
The role of eyes in early face processing: A rapid adaptation study of the inversion effect
}

\author{
Dan Nemrodov and Roxane J. Itier ${ }^{\star}$ \\ University of Waterloo, Waterloo, Canada
}

\begin{abstract}
The current study employed a rapid adaptation procedure to test the neuronal mechanisms of the face inversion effect (FIE) on the early face-sensitive event-related potential (ERP) component N170. Five categories of face-related stimuli (isolated eyes, isolated mouths, eyeless faces, mouthless faces, and full faces) and houses were presented in upright and inverted orientations as adaptors for inverted full face test stimuli. Strong adaptation was found for all face-related stimuli except mouths. The adaptation effect was larger for inverted than upright stimuli, but only when eyes were present. These results underline an important role of eyes in early face processing. A mechanism of eye-dependent orientation sensitivity during the structural encoding stage of faces is proposed.
\end{abstract}

For the past 25 years, face perception research has been largely influenced by the cognitive architecture model developed by Bruce and Young (1986). This model stipulates that in a first step, facial features and configural information (the relationships between these features) are processed through a structural encoding mechanism from which information is extracted to allow the analysis of emotional expressions, facial speech, identity, and other visual processes. However, the exact nature of this encoding mechanism and its neural underpinnings remain unclear. The present paper argues that eyes play a crucial role in this structural encoding process at the neural level.

Much of the research on early face processing has been centred around the face-sensitive scalp event related potential (ERP) N170 component (e.g., Bentin, Allison, Puce, Perez, \& McCarthy, 1996; Rossion \& Jacques, 2008) and its magnetic counterpart M170 (e.g., Halgren, Raij, Marinkovic, Jousmaki, \& Hari, 2000; Harris \& Nakayama, 2008; Itier, Herdman, George, Cheyne, \& Taylor, 2006). The N170 component is maximally recorded over lateral temporo-occipital scalp sites between 150 and $190 \mathrm{~ms}$ after face onset and exhibits stronger sensitivity to faces or eyes (but usually not other face parts) than to objects (Itier, Latinus, \& Taylor, 2006; Itier \& Taylor, 2004; Rossion et al., 2000). This component is viewed as a marker of the early stages of face processing representing the structural encoding mechanism of Bruce and Young's (1986) model (Eimer, 2000b; Rossion, Campanella et al., 1999).

One of the hallmarks of face specialness is the face inversion effect (FIE), a disproportional disruption of upside down (inverted) face perception and recognition compared to inverted objects (Yin, 1969). This effect has been interpreted as reflecting the impairment of configural processing characteristic of faces, in favour of feature-based processing typical of objects (Farah, Wilson, Maxwell Drain, \& Tanaka, 1995). Inversion of faces also triggers an 
increase in amplitude and latency of N170 (Bentin et al., 1996; Eimer, 2000a; Itier \& Taylor, 2002; Rossion, Campanella et al., 1999). This early inversion effect, one of the most robust findings in the face ERP literature, is much larger for faces than other objects, which often display no inversion effect at all, or an 'inverted' inversion effect with smaller amplitudes for inverted than upright items (Itier, Alain, Sedore, \& McIntosh, 2007; Itier, Latinus et al., 2006; Rossion et al., 2000).

This increase in amplitude and latency of the N170 with face inversion has been interpreted by some as reflecting the response of neurons sensitive to non-facial object stimuli in addition to the face-sensitive cell response (Haxby et al., 1999; Rossion \& Gauthier, 2002; Yovel \& Kanwisher, 2005). That is, by disrupting the face configuration, inversion would force the system to process inverted faces in a feature-based manner, involving objectsensitive neurons to do so. However, this interpretation is at odds with the strong attenuation of the FIE following removal of the eyes from the face stimuli (Itier et al., 2007; Itier, Van Roon, \& Alain, 2011). In addition, isolated eyes do not show the FIE but induce, either in upright or inverted orientation, an N170 amplitude roughly similar to that induced by inverted faces (Itier et al., 2007; Itier, Latinus et al., 2006). Based on these results, Itier and colleagues proposed that the FIE is mostly driven by the presence of the eyes. They developed a neural model of early face processing accounting for the various modulations of the N170 component reported in the literature (Itier et al., 2007; Itier \& Batty, 2009). The model is based on human intracranial data (Allison, Puce, Spencer, \& McCarthy, 1999; McCarthy, Puce, Belger, \& Allison, 1999; Puce, Allison, \& McCarthy, 1999) and single unit recording data in monkeys (Perrett, Rolls, \& Caan, 1982; Perrett et al., 1985) and posits that face-sensitive and eye-sensitive neurons coexist in the human brain and that their response modulations account for the variations in N170 amplitude. Based on plausible cell properties, both face and eye-sensitive neurons respond to isolated eyes, but only facesensitive neurons respond to faces (Figure 1). In addition, the model posits that eye-sensitive neurons do not respond to the eyes of the face due to a possible inhibition mechanism exacted by face-sensitive neurons onto eye-sensitive neurons or some other inactivation mechanism, assumed to take place because of the configural face context. As a result, facesensitive neurons response dominates during the presentation of upright faces and does not change when eyes are removed from the face (Eimer, 1998; Itier et al., 2007, 2011).

However, when a face is inverted the inhibition/inactivation mechanism stops, allowing eyesensitive neurons to respond to eyes, which along with the response of face-sensitive neurons results in the classic increase in N170 amplitude for inverted compared to upright faces. Similarly, the presentation of isolated eyes triggers the response of eye-sensitive neurons in addition to face-sensitive neurons, leading to roughly similar N170 amplitudes for eyes and inverted faces, that is, larger than upright faces (Bentin et al., 1996; Itier et al., 2007; Jemel, George, Chaby, Fiori, \& Renault, 1999; Taylor, Itier, Allison, \& Edmonds, 2001). Finally, the model accounts for the strong reduction or even lack of FIE reported for eyeless faces (Itier et al., 2007, 2011), as eye-sensitive neurons are only active in the presence of eyes.

To further investigate the nature of the FIE on N170, a recent study employed a rapid adaptation technique involving brief stimulus presentations with a short inter-stimulus interval (Eimer, Kiss, \& Nicholas, 2010). The adaptation technique relies on the activity reduction of neuronal populations in response to a test stimulus (S2) immediately preceded by a categorically similar adaptor stimulus ( $\mathrm{S} 1)$. This adaptation paradigm has been successfully implemented in fMRI (e.g., Grill-Spector, Henson, \& Martin, 2006), ERP (e.g., Caharel, d'Arripe, Ramon, Jacques, \& Rossion, 2009; Kovács et al., 2006) and magnetoencephalography (MEG) studies (e.g., Harris \& Nakayama, 2007). In contrast to previous ERP adaptation studies that used only upright faces as S2 (Caharel et al., 2009; Kovács et al., 2006), Eimer et al. (2010) investigated the adaptation to upright faces, inverted faces, 
eyeless faces, and isolated eye regions stimuli. This allowed them to address two key tenets of the model by Itier et al. (2007 - hereafter the Itier et al. model): the inhibition of eyesensitive neurons in response to upright face presentation and their involvement in the N170 FIE.

Eimer et al. (2010) reasoned that if eye-sensitive neurons were inhibited by face-sensitive neurons during the processing of upright faces, then upright faces and upright eyeless faces would be functionally equivalent in terms of N170 adaptation effects. In other words, when presented as S1 adaptors both stimuli should produce the same level of adaptation for all test stimuli. In addition, they should also exhibit the same pattern of N170 adaptation across different adaptor categories when presented as S2 test stimuli. According to the Itier et al. model, inversion of a face releases the eye-sensitive neurons from inhibition so that both eye and face-sensitive neurons respond to inverted faces, like they do for isolated eyes.

Therefore, Eimer et al. predicted that, for both isolated eyes and inverted face test stimuli, isolated eyes and inverted face adaptors would provide stronger adaptation than eyeless face or upright face adaptors (which elicit response from face-sensitive neurons only).

The results of Eimer et al. (2010) study provided partial support for the Itier et al. model. As predicted, for eye test stimuli, eye and inverted face adaptors produced stronger adaptation than upright full face and eyeless face adaptors for which no difference was seen. Overall, there was no amplitude difference between upright face and upright eyeless face stimuli either as adaptor or test stimuli. However, in contradiction with the model's prediction, for inverted face test stimuli, eye adaptors did not trigger stronger adaptation than either upright faces or upright eyeless faces. The results of this study also provided evidence against the involvement of object-sensitive neurons in the FIE. Indeed, if object-sensitive neurons were involved in the processing of inverted faces, one would predict that objects such as houses presented as adaptors should diminish the inversion effect because object-sensitive neurons would respond less to the face test stimuli. However, Eimer et al. (2010) showed that presenting houses as adaptors failed to diminish the FIE (i.e., the difference between upright and inverted test stimuli) compared to presenting upright faces as adaptors. The authors concluded that for upright face test stimuli the adaptation was determined by the category of the preceding adaptor, whereas for inverted face stimuli the adaptation was also dependent on the adaptor orientation. The authors suggested the FIE could reflect both a category and an orientation-sensitive mechanism.

In the present study, we tried to replicate and extend Eimer et al. (2010) findings obtained with inverted face test stimuli. We used the same rapid adaptation procedure where an adaptor stimulus (S1) and a subsequent test stimulus (S2) were both presented for $200 \mathrm{~ms}$ and separated by short intervals. Adaptation effects were measured for the N170 component in response to inverted faces only (S2), according to each adaptor category. Please note that although effects of face adaptation were recently reported for later ERP components (e.g., Burkhardt et al., 2010; Kloth \& Schweinberger, 2010), the present study focussed on rapid adaptation effects on the N170 exclusively. In order to test Eimer et al.'s suggestion that the N170 FIE is mediated by orientation-sensitive neurons (hereafter orientation-sensitive hypothesis or $\mathrm{OSH}$ ), each stimulus category used in the original paper was presented in both upright and inverted orientation. Thus, in addition to the upright and inverted full faces, and upright houses, eyes, and eyeless faces tested by Eimer et al. (2010), we included inverted houses, inverted eyeless faces, and inverted isolated eyes as adaptors. Furthermore, in order to ascertain the special role of eyes in the FIE as predicted by the Itier et al. model, isolated mouths and mouthless faces were included in the design, both upright and inverted. Like eyes, mouths are also an isolated facial feature. Mouthless faces control for the configuration disruption involved in eyeless faces while keeping the eyes within the face. 
According to the $\mathrm{OSH}$, larger adaptation effects should be found for inverted than upright adaptors regardless of the category. Thus, in response to $\mathrm{S} 2$, a decreased activation should be found for inverted compared to upright adaptors, for each adaptor category tested: houses, mouths, eyes, faces, eyeless faces, and mouthless faces. Furthermore, this hypothesis does not make any claim regarding the special role of eyes in the FIE and thus no adaptation advantage should be found for isolated eyes over upright faces or upright eyeless faces. According to the Itier et al. model, inverted faces and isolated eyes recruit both face and eye-sensitive neurons while upright faces and upright and inverted eyeless faces recruit only face-sensitive neurons. This model thus predicts maximal adaptation for inverted faces and isolated eyes adaptors. However, there should not be larger adaptation for inverted than upright eyeless faces and isolated eye adaptors. The model also predicts no difference in adaption between eyeless faces (upright or inverted) and upright full faces. If eyes are special, they should elicit a different response compared to other facial features like mouths, starting at the S1 level. The adaptation for eyes should also be different from the adaptation for mouths. Mouthless faces in contrast, should behave like full faces despite the slightly disrupted facial configuration, but not like eyeless faces that are missing the critical eye feature. That is, inverted mouthless faces should produce the same adaptation as inverted full faces.

\section{Materials and methods}

\section{Participants}

Eighteen participants were paid $\$ 10 / \mathrm{h}$ or participated for course credits and gave signed, informed consent as approved by the Research Ethic Board of the University of Waterloo before testing began. Subjects had normal or corrected-to-normal visual acuity. Three participants were excluded from the final analysis due to low accuracy rate or an insufficient number of trials per condition after artefact rejection. The remaining 15 participants (7 males) were 19-43 years old (mean age 22.1 years). Fourteen were right handed, and one was left handed.

\section{Stimuli and procedure}

Stimuli consisted of faces, eyeless faces, mouthless faces, mouths alone, eyes alone, and houses presented upright and inverted, adapted from Itier et al. (2007), as well as flowers (13 categories total). Faces were of Caucasian individuals, displaying neutral expression and showing part of the hair. Isolated eyes were created by cropping the eye region (including eye brows) from faces so that the centre of the rectangular eye region was approximately the centre of the eyes. Isolated mouths were created by cropping out a rectangle around the mouth. Eyeless and mouthless faces were created by erasing the eye or mouth region and filling it with skin-like texture. The images were equated in luminance $\left(48 \mathrm{~cd} / \mathrm{m}^{2}\right)$ and contrast by adding pixelized background noise. Then the images' rotational average of the Fourier amplitude spectrum content was matched over all images using SHINE (Spectrum, Histogram, and Intensity Normalization and Equalization) toolbox (Willenbockel et al., 2010, www.mapageweb.umontreal.ca/gosselif/shine), so that spatial frequencies were also equated.

Subjects sat in a dimly lit room $60 \mathrm{~cm}$ in front of a computer 17" Cathode Ray Tube (CRT) monitor. Presentation software (Neurobehavioral Systems, http://www.neurobs.com) was used for stimulus presentation and behavioural response collection recorded from a Nintendo game controller. In each trial, participants were presented with a pair of images in quick succession. They were instructed to press one button if either the first or the second picture was a sunflower and another button if no sunflower was shown. They were required 
to make their button press following the second image presentation. Response buttons were counterbalanced across participants.

Each S1 stimulus category included 44 different images presented twice. On each trial, two images (S1: adaptor stimulus; S2: test stimulus) were presented successively for $200 \mathrm{~ms}$ each, separated by one of three equiprobable intervals of 234, 250, and $267 \mathrm{~ms}$, to reduce anticipation of S2. Participants were given on average 1,500 ms to respond (range 1,300$1,700 \mathrm{~ms}$ ) before the next stimulus came on. Only inverted faces and flowers served as S2. During the experiment, 88 pictures were thus presented for each S1 category, 8 of which were followed by a sunflower and thus excluded from further analysis. In addition, sunflowers appeared 96 times as S1 stimuli and these trials were also excluded from further analysis. The appearance of the sunflower image as S1 or S2 was equiprobable. In total, of the 1,152 trials presented during the experiment, 960 trials contained no sunflower and were retained for analysis ( 80 trials for each of the 12 categories). All images were presented in random order in four blocks (of 264 pairs of stimuli, 240 containing no flowers and 24 pairs of stimuli containing flowers either as S1 or S2), with self-paced breaks between them.

\section{EEG recording and data analysis}

The electroencephalogram (EEG) was recorded from $64 \mathrm{Ag} / \mathrm{AgCl}$ active electrodes (BioSemi Active Two system, the Netherlands: http://www.biosemi.com) mounted on a flexible cap according to the extended international 10/20 systems. A Common Mode Sense (CMS) active electrode and Driven Right Leg (DRL) passive electrode serving as ground were used during acquisition. Eight additional electrodes were used: two electrodes were placed on the posterior part of the cap on the left and right sides (CB1 and CB2, respectively). Four additional electrodes served to record horizontal and vertical eye movements and were placed at the outer canthus and under the centre of each eye. Two more electrodes were placed on the left and right mastoids (TP9 and TP10). EEG was digitized at a sampling rate of $512 \mathrm{~Hz}$. The data were processed using the EEGLab toolbox (Delorme \& Makeig, 2004) implemented in Matlab (Mathworks, Inc., Natick, MA). Only correct-response trials were analysed. EEG was epoched offline from $100 \mathrm{~ms}$ before S1 onset (baseline) to $400 \mathrm{~ms}$ after S2 onset. Trials were then digitally band-pass filtred (0.01$30 \mathrm{~Hz})$ and average referenced. Trials containing artefacts $(> \pm 65 \mu \mathrm{V})$ and trials with abnormal trends (exceeding maximal slope of $50 \mu \mathrm{V}$ with $\mathrm{R} 2$ limit $=0.3$ ) were then rejected. Ocular artefacts were first removed using independent component analysis (ICA) decomposition as implemented in EEGLab. On average 69.2 (range: 58-80) trials were retained in each condition for each subject. The EEG waveforms were averaged separately for each category according to the two stimuli: once locked on S1 and relative to a $100 \mathrm{~ms}$ pre-S1 baseline $(-100 \mathrm{~ms}$ to $+400 \mathrm{~ms}$ from $\mathrm{S} 1)$ and once locked on $\mathrm{S} 2$ and relative to a 50 $\mathrm{ms}$ pre-S2 and $50 \mathrm{~ms}$ post-S2 baseline as done by Eimer et al. (2010) (-50 ms to $+400 \mathrm{~ms}$ from S2). N170 components in response to S1 or S2 stimuli were quantified within a 150250-ms time interval after stimulus onset. For S1 and S2, N170 was measured at lateral posterior electrodes CB1 (left hemisphere) and CB2 (right hemisphere) where the inversion effect for full faces as well as the adaptation effects, were maximal.

Repeated-measures analyses of variance (ANOVAs) were performed on N170 peak amplitudes with the factors adaptor category (6), adaptor orientation (2), and laterality (2) in both S1 and S2 analyses. N170 latencies were also analysed for S1. When the adaptor category by adaptor orientation interaction was significant a further repeated-measures ANOVA was performed for each orientation separately to test for category effects (i.e., adaptor category $(6) \times$ laterality $(2))$ and for each adaptor category separately to test for orientation effects (i.e., adaptor orientation $(2) \times$ laterality $(2))$. For all analyses, 
Greenhouse-Geisser corrections to the degrees of freedom were performed where appropriate. Bonferroni correction was used for multiple comparisons.

\section{Results}

\section{Behavioural analysis}

We analysed the correct rejection rate for $\mathrm{S} 2$ as a function of $\mathrm{S} 1$ adaptors, using a 2 (adaptor orientation) $\times 6$ (adaptor categories) ANOVA. Average correct rejection accuracy for the test stimuli was $91.3 \%$. No main effects were found. Although a small interaction between adaptor orientation and adaptor categories was found $\left(F(5,70)=2.87, p=.021, \eta^{2}=0.17\right)$, post hoc comparisons did not yield any significant differences between conditions. In other words, the various adaptor categories had no specific effect on S2 discrimination.

\section{ERP-S1 analysis}

For the N170 peak amplitude, no main effect of and no interaction with laterality were found. The main effects of adaptor orientation $\left(F(1,14)=9.84, p=.007, \eta^{2}=0.41\right)$ and adaptor category $\left(F(5,70)=27.88, p<.001, \eta^{2}=0.67\right)$, and the adaptor orientation $\times$ adaptor category interaction $\left(F(5,70)=8.61, p=.001, \eta^{2}=0.31\right)$ were significant. The larger amplitude for all face-related stimuli, including isolated mouths, compared to houses $(p<.01)$ confirmed the face sensitivity of the N170 component (see Figure 2). When upright adaptor categories were analysed separately (main effect of adaptor category, $F=$ $21.7, p<.0001$ ), eyes yielded the largest N170 amplitudes although paired comparisons were significant only with houses $(p<.0001)$ and eyeless faces $(p<.008)$, with a trend seen with full faces $(p=.06)$. In contrast, no difference was seen between upright full faces, eyeless faces, mouthless faces, and isolated mouths. Houses yielded the smallest amplitudes ( $p<.05$ or less for all comparisons). When inverted adaptor categories were analysed separately (main effect of adaptor category, $F=27.6, p<.0001$ ), paired comparisons revealed that amplitudes for inverted faces no longer differed from that of eyes, but were larger than for mouths $(p=.013)$ and tended to be larger than for eyeless faces $(p=.07)$. Each adaptor category was also analysed separately to test for inversion effects (see Figure $3)$. No inversion effect was found for houses, mouths, or, eyes while the classic FIE was found for faces $(F(1,14)=19.32, p<.001)$, mouthless faces $(F(1,14)=12.34, p<.005)$ but also eyeless faces $(F(1,14)=6.11, p<.05)$, with larger amplitudes for inverted than upright stimuli (see Figure 2).

For the N170 latency, a main effect of category $\left(F(5,70)=16.67, p<.001, \eta^{2}=0.54\right)$ was found. The N170 was much delayed for isolated mouths than for all the other categories $(p$ $<.005$ for all comparisons). As expected, N170 for isolated eyes peaked later than for faces $(p=.003)$ or mouthless faces $(p=.013)$, which did not differ. N170 was also delayed for eyeless faces compared to normal faces $(p<.001)$ but no difference was seen between faces and houses. A main effect of orientation $\left(F(1,14)=18.71, p=.001, \eta^{2}=0.57\right)$ was also found, due to delayed latencies for inverted compared to upright categories.

\section{ERP-S2 analysis}

The analysis of N170 peak amplitude revealed neither a main effect of, nor an interaction with, laterality. The main effects of adaptor orientation $\left(F(1,14)=4.85, p<.05, \eta^{2}=0.25\right)$ and adaptor category $\left(F(5,70)=31.26, p<.0001, \eta^{2}=0.69\right)$, and the adaptor orientation $\times$ adaptor category interaction $\left(F(5,70)=6.29, p=.001, \eta^{2}=0.31\right)$ were significant. When upright categories were analysed separately, a main effect of adaptor category was found $(F(1,14)=84, p<.0001)$. Post hoc comparisons revealed that amplitudes were largest for houses compared to any other category but no differences were found between any of the other categories. When inverted categories were analysed separately, the main effect of 
adaptor category $(F(1,14)=42.09, p<.0001)$ was also significant. Paired comparisons revealed larger amplitudes for houses than all other categories $(p<.0001)$ except mouths for which a trend was found $(p=.07)$. Amplitudes for mouths were also larger than other facerelated stimuli $(p<.005)$. However, no differences were found between eyes, faces, eyeless faces, and mouthless faces.

Adaptor categories were also analysed separately and a main effect of adaptor orientation was found for eyes $(F(1,14)=9.7, p<.01)$, faces $(F(1,14)=6.2, p<.05)$ and mouthless faces $(F(1,14)=6.7, p<.05)$, with smaller amplitudes (and so larger adaptation effects) for inverted than upright adaptor categories (Figures 4-5). The inversion effect was also significant for mouths $(F(1,14)=5.3, p<.05)$ but in the opposite direction with larger amplitudes (and smaller adaptation effect) for inverted than upright mouths. No inversion effect was found for houses $(p=.18)$ and it was borderline significant for eyeless faces $(F(1,14)=4.5, p=.053)$. It is important to note that when the difference between P1 and N170 peak was calculated, the inversion effect disappeared completely $(p>.5)$ for eyeless faces but remained unchanged for all the other categories.

\section{Discussion}

The nature of the FIE and in particular whether it stems from a quantitative change in the activity of face-sensitive neurons or from the recruitment of qualitatively different neuronal population (e.g., object-sensitive neurons) is still very controversial (Haxby et al., 1999; Rossion, Delvenne et al., 1999; Sadeh \& Yovel, 2010; Watanabe, Kakigi, \& Puce, 2003; Yovel \& Kanwisher, 2005). The present study employed a rapid adaptation procedure to study the FIE on the N170 ERP component. The experiment was designed to test the predictions of the Itier et al. model and the orientation-sensitive neuronal hypothesis $(\mathrm{OSH})$ proposed recently by Eimer et al. (2010). The Itier et al. model stipulates that the FIE hinges mainly upon the response of eye-sensitive neurons (in addition to face-sensitive neurons) to the presentation of inverted face stimuli because the disruption of the face configuration by inversion would release the eye-sensitive neurons from their inhibition by face-sensitive neurons. In contrast, the OSH assumes that the FIE is mediated by neurons' orientation sensitivity. To test these predictions, the current study replicated the design of the rapid adaptation study conducted by Eimer et al. (2010) using various face stimuli adaptors presented upright and upside down and inverted face test stimuli.

The results of the N170 analysis for the adaptor stimuli (S1) revealed a customary increase of the amplitude for face-related stimuli compared to houses, even for isolated mouths. Eyes yielded the largest amplitudes as generally reported (Bentin et al., 1996; Itier et al., 2007; Itier, Latinus et al., 2006; Taylor et al., 2001) and the classical FIE was found for faces and mouthless faces, but was not present for eyes, mouths, and houses and was attenuated (although significant) for eyeless faces.

Similarly to Eimer et al. (2010), the analysis of S2 amplitude revealed a broad adaptation effect elicited by face-related adaptors compared to houses, although to a lesser extent by mouths compared to the other face stimuli. We also found a larger adaptation for inverted compared to upright faces as also reported by Eimer et al. (2010). In line with the predictions of the Itier et al. model, a similar adaptation was found for inverted and upright eyeless faces (although the effect of orientation was borderline), which did not differ from that of upright full faces. This result provides support to the idea that the FIE is somehow related to the presence of eyes. However, contrary to the model prediction, an inversion effect was found for eyes which, when upright, did not yield a similar adaptation effect as inverted faces, a result also obtained by Eimer et al. (2010). When presented inverted 
however, adaptation for eyes and faces did not differ. These results suggest that another factor, previously not accounted for by the Itier et al. model, is involved in the FIE.

To account for this difference between eyes and inverted face adaptation to inverted face test stimuli as well as for their result of higher adaptation for inverted than upright houses, Eimer et al. (2010) suggested that face-sensitive neurons may be tuned to orientation (OSH). One difficulty with this hypothesis, acknowledged by the authors, is the dissociation between the differential adaptation effects triggered by inverted compared to upright houses on S2 and a lack of differential response of N170 between inverted and upright houses presented as S1 that was found in that study. To explain this discrepancy, Eimer et al. (2010) suggested an input from the areas responsible for non-face object processing onto the orientation-tuned face-sensitive neurons whenever objects in a non-canonical orientation are encountered. This hypothesis, however, cannot fully account for the results obtained in the current study. According to the OSH, neurons involved in face processing are orientation sensitive and are responding more for inverted than upright facial stimuli. We thus expected to have an adaptation advantage for all inverted stimuli, facial stimuli due to an orientation tuning of face-sensitive neurons, and non-facial stimuli due to the proposed input from areas dedicated to non-face object processing. In contrast, we found no inversion advantage on the adaptation response to houses and isolated mouths (for which an adaptation advantage was found when presented upright), and an attenuation of this inversion advantage for faces lacking the eye region.

In view of the present results, we want to propose a more nuanced account. Our N170 results underline the important role of eyes in modulating the adaptation effect. Based on our results using several inverted adaptors with or without eyes, we may conclude that the orientation sensitivity of the neuronal population generating the N170 is at least partially dependent on the presence of the eye region. Single-cell studies in monkeys have shown that some face-sensitive cells in inferior temporal (IT) cortex and superior temporal sulcus (STS) where they are prevalent, are often sensitive not only to specific face features, but also to a specific view point (Perrett, Hietanen, Oram, Benson, \& Rolls, 1992; Perrett et al., 1991). In addition, many neurons in these areas tend to have large receptive fields of up to $25^{\circ}$ squared, which would allow them to integrate spatially separated features (Desimone, Albright, Gross, \& Bruce, 1984; Freiwald, Tsao, \& Livingstone, 2009; Perrett et al., 1985; Tsao \& Livingstone, 2008), although some studies suggest that they retain a sensitivity to small position changes (Op De beeck \& Vogels, 2000 - for a more detailed discussion, see Rousselet, Thorpe, \& Fabre-Thorpe, 2004). A recent study found that cells in the middle face patch of monkey inferotemporal cortex were mostly responsive to face parts and their combinations, with a focus on features related to the eyes and the face outline geometry (Freiwald et al., 2009). Furthermore, the comparison of their response to upright and inverted faces showed that the identity of individual features was interpreted according to the metrics of an upright face template, that is, these cells were tuned to the specific position of the features within the face. These neuronal qualities would be ideal to code feature orientation in relation to other facial features, which itself would code for the whole face orientation. Here, we propose that eyes act as an anchor point or reference point from which the position and orientation of other features are coded in order to generate a face percept.

If eyes were to serve as a reference point for the orientation sensitivity of neurons, we would expect neuronal activity to be relatively unaffected by the orientation of eyes, which is consistent with the general lack of inversion effect reported on the N170 for isolated eyes (Itier et al., 2007; Itier, Latinus et al. 2006; Itier et al., 2011). Both monkeys (Gothard, Brooks, \& Peterson, 2009) and humans show a strong reliance on the eye region when processing upright as well as inverted faces (Sekuler, Gaspar, Gold, \& Bennett, 2004; Williams \& Henderson, 2007). In addition, if eyes were to serve as a reference point for 
neurons orientation sensitivity, it would be essential that they would be processed first during the time course of face encoding. In line with this expectation, Schyns and colleagues using EEG and the Bubble technique, have shown that sensitivity to facial features starts approximately $50 \mathrm{~ms}$ prior to the N170 peak with encoding of the left eye (Schyns, Petro, \& Smith, 2007; Schyns, Petro, \& Smith, 2009). Together, these results support the hypothesis that eyes represent a reference for perception of other facial features and face configuration, which could be achieved through the modulation of face-sensitive neurons' activity by eyesensitive neurons. The neurophysiological feasibility of this mechanism is supported by single unit recording evidence that the eye region provides an excitatory input to the cells responding to full faces (Perrett et al., 1985). Thus, the orientation tuning of the neurons proposed by Eimer et al. (2010) would not be in absolute gravitational terms but in reference to eyes and this could be achieved by modulatory input from eye-sensitive neurons onto face-sensitive neurons.

The original model by Itier et al. (2007) stipulates that face-sensitive neurons respond to isolated eyes and can decrease the activity of eye-sensitive neurons (inhibition) in response to upright faces. The model also posits that the disruption of the face configuration by inversion releases the eye-sensitive neurons from their inhibition. Eimer et al. (2010) showed that, like upright face adaptors, eye adaptors yielded maximum adaptation to upright face test stimuli, which supports the idea that face-sensitive neurons respond to eyes. They also showed a larger adaptation effect for inverted than upright face adaptors to isolated eye test stimuli, in line with the hypothesis of the release from inhibition of eye-sensitive neurons. However, the present results of a larger adaptation for inverted than upright isolated eye adaptors to inverted faces are not in line with the model's prediction. Here we propose that eye-sensitive neurons can also modulate the activity of face-sensitive neurons, thus allowing eye-anchored orientation sensitivity. This new feature of the model together with our proposed orientation tuning sensitivity relative to the eyes (rather than absolute) can, in our view, explain the pattern of results obtained in the Eimer et al. (2010) and the current studies. Thus, while the increased N170 amplitude with face inversion may still be the result of the eye-sensitive neurons activation in addition to the face-sensitive neuron activation as proposed by Itier et al. (2007), we now propose that the face-sensitive neuron activation may be different for upright and inverted faces. The idea that different cell populations code for upright and inverted faces was also discussed by Eimer et al. (2010) and has been supported by simultaneous opposite aftereffects for upright and inverted faces (Rhodes et al., 2004). However here, we propose that the coding of upright and inverted faces is related to the eyes. That is, the neurons maximally tuned to the information beneath the eyes would be active in response to upright faces while neurons maximally tuned to the information above the eyes would be active in response to inverted faces. In other words, different face-sensitive neurons would respond according to the position of the facial features in relation to the eyes. This differential neuronal recruitment may also account for different patterns of adaptation obtained in fMRI studies, which show higher discrimination between same and different upright faces compared to same and different inverted faces (Gilaie-Dotan, Gelbard-Sagiv, \& Malach, 2010; Yovel \& Kanwisher, 2005), as neurons responding to upright faces may be more involved in processing face identity and other facerelated attributes than inversion-tuned face-sensitive neurons.

This possible eye-anchored mechanism would be in line with other results indicating an important role of eyes in modulating the adaptation effects found in the present study. For example, it can provide an explanation for the discrepancy between the lack of inversion effect on N170 amplitude for eyes (Itier et al., 2007; Itier, Latinus et al., 2006; Itier et al., 2011), also found in the present study for S1 stimuli, and the inversion effect obtained in the adaptation results with eyes as adaptors. Although both upright and inverted eyes activate eye-sensitive neurons, only inverted eyes would modulate the inversion-tuned face-sensitive 
neurons thus creating a larger adaptation response to the inverted face. However, for eyes presented at S1, such difference would not be expected if we assume that upright-tuned and inversion-tuned face-sensitive neuronal populations produce roughly similar activation and thus similar N170 amplitudes. Finally eyes, although the most important of all facial features, may not be the only anchoring feature. In previous studies, eyeless faces or faces with no clearly visible eyes like Mooney faces elicited no or strongly attenuated FIE (Itier $e t$ al., 2007, 2011; Latinus \& Taylor, 2005; Sagiv \& Bentin, 2001). In contrast, in the present study, at the S1 level the inversion of eyeless faces produced a significant FIE, which suggests the orientation tuning mechanism can be activated in the absence of eyes. This discrepancy with previous studies could be related to the adaptation paradigm although this remains to be determined in future studies.

In conclusion, the results of the present study are consistent with the principles proposed in the Itier et al. model concerning the modulation of eye-sensitive neurons' activity by facesensitive neurons in response to upright faces (possible inhibition mechanism). They also partially support the OSH proposed by Eimer et al. (2010) by demonstrating orientationsensitive adaptation for stimuli containing eyes. However, the attenuation of this sensitivity for eyeless stimuli suggests a possible modulation of the activity of face-sensitive neurons by eye-sensitive neurons, a possibility not accounted for by the Itier $e t$ al. model. Therefore, we suggest a mechanism in which eyes act as an anchor point from which the position and orientation of other features are coded in order to generate a face percept. This explanation is of course speculative and the exact nature of the neuronal populations recruited in these effects will have to be tested in future studies. The results of the present study underline again the importance of eyes in early face processing, an aspect underplayed in the original Bruce and Young (1986) model. However, the exact role of eyes in these early structural encoding stages of face processing remains to be empirically determined (for extended discussion, see Itier \& Batty, 2009).

\section{Acknowledgments}

This research was funded by the Canadian Institutes for Health Research (CIHR, MOP-89822), the Ontario Research Fund (ORF), the Canada Foundation for Innovation (CFI), and the Canada Research Chair (CRC) program to RJI.

\section{References}

Allison T, Puce A, Spencer DD, McCarthy G. Electrophysiological studies of human face perception. I: Potentials generated in occipitotemporal cortex by face and non-face stimuli. Cerebral Cortex. 1999; 9:415-430.10.1093/cercor/9.5.415 [PubMed: 10450888]

Bentin S, Allison T, Puce A, Perez E, McCarthy G. Electrophysiological studies of face perception in humans. Journal of Cognitive Neuroscience. 1996; 8:551-565.10.1162/jocn.1996.8.6.551 [PubMed: 20740065]

Bruce V, Young A. Understanding face recognition. British Journal of Psychology. 1986; 77:305-327. [PubMed: 3756376]

Burkhardt A, Blaha LM, Jurs BS, Rhodes G, Jeffery L, Wyatte D, Busey T. Adaptation modulates the electrophysiological substrates of perceiving facial distortion: Support for opponent coding. Neuropsychologia. 2010; 48:3743-3756.10.1016/j.neuropsychologia.2010.08.016 [PubMed: 20736026]

Caharel S, d'Arripe O, Ramon M, Jacques C, Rossion B. Early adaptation to repeated unfamiliar faces across viewpoint changes in the right hemisphere: Evidence from the N170 ERP component. Neuropsychologia. 2009; 47:639-643.10.1016/j.neuropsychologia.2008.11.016 [PubMed: 19084547]

Desimone R, Albright TD, Gross CG, Bruce C. Stimulus-selective properties of inferior temporal neurons in the macaque. The Journal of Neuroscience. 1984; 4:2051-2062. [PubMed: 6470767] 
Delorme A, Makeig S. EEGLAB: An open source toolbox for analysis of single-trial EEG dynamics including independent component analysis. Journal of Neuroscience Methods. 2004; 134:921.10.1016/j.jneumeth.2003.10.009 [PubMed: 15102499]

Eimer M. Does the face-specific N170 component reflect the activity of a specialized eye processor? Neuroreport. 1998; 9:2945-2948.10.1097/00001756-199809140-00005 [PubMed: 9804295]

Eimer M. Effects of face inversion on the structural encoding and recognition of faces: Evidence from event-related brain potentials. Cognitive Brain Research. 2000a; 10:145-158.10.1016/ S0926-6410(00)00038-0 [PubMed: 10978702]

Eimer M. The face-specific N170 component reflects late stages in the structural encoding of faces. Neuroreport. 2000b; 11:2319-2324. [PubMed: 10923693]

Eimer M, Kiss M, Nicholas S. Response profile of the face-sensitive N170 component: A rapid adaptation study. Cerebral Cortex. 2010; 20:2442-2452.10.1093/cercor/bhp312 [PubMed: 20080930]

Farah J, Wilson KD, Maxwell Drain H, Tanaka R. The inverted face inversion effect in prosopagnosia: Evidence for mandatory, face-specific perceptual mechanisms. Vision Research. 1995; 35:20892093.10.1016/0042-6989(94)00273-O [PubMed: 7660612]

Freiwald WA, Tsao DY, Livingstone MS. A face feature space in the macaque temporal lobe. Nature Neuroscience. 2009; 12:1187-1196.10.1038/nn.2363

Gilaie-Dotan S, Gelbard-Sagiv H, Malach R. Perceptual shape sensitivity to upright and inverted faces is reflected in neuronal adaptation. NeuroImage. 2010; 50:383-395.10.1016/j.neuroimage. 2009.12.077 [PubMed: 20044007]

Gothard KM, Brooks KN, Peterson A. Multiple perceptual strategies used by macaque monkeys for face recognition. Animal Cognition. 2009; 12:155-167.10.1007/s10071-008-0179-7 [PubMed: 18787848]

Grill-Spector K, Henson R, Martin A. Repetition and the brain: Neural models of stimulus-specific effects. Trends in Cognitive Sciences. 2006; 10:14-23.10.1016/j.tics.2005.11.006 [PubMed: 16321563]

Halgren E, Raij T, Marinkovic K, Jousmaki V, Hari R. Cognitive response profile of the human fusiform face area as determined by MEG. Cerebral Cortex. 2000; 10:69-81.10.1093/cercor/ 10.1.69 [PubMed: 10639397]

Harris A, Nakayama K. Rapid face-selective adaptation of an early extrastriate component in MEG. Cerebral Cortex. 2007; 17:63-70.10.1093/cercor/bhj124 [PubMed: 16436684]

Harris A, Nakayama K. Rapid adaptation of the M170 response: Importance of face parts. Cerebral Cortex. 2008; 18:467-476.10.1093/cercor/bhm078 [PubMed: 17573371]

Haxby V, Ungerleider LG, Clark VP, Schouten L, Hoffman EA, Martin A. The effect of face inversion on activity in human neural systems for face and object perception. Neuron. 1999; 22:189199.10.1016/S0896-6273(00)80690-X [PubMed: 10027301]

Itier RJ, Alain C, Sedore K, McIntosh AR. Early face processing specificity: It's in the eyes! Journal of Cognitive Neuroscience. 2007; 19:1815-1826.10.1162/jocn.2007.19.11.1815 [PubMed: 17958484]

Itier RJ, Batty M. Neural bases of eye and gaze processing: The core of social cognition. Neuroscience and Biobehavioral Reviews. 2009; 33:843-863.10.1016/j.neubiorev.2009.02.004 [PubMed: 19428496]

Itier RJ, Herdman AT, George N, Cheyne D, Taylor MJ. Inversion and contrast-reversal effects on face processing assessed by MEG. Brain Research. 2006; 1115:108-120.10.1016/j.brainres. 2006.07.072 [PubMed: 16930564]

Itier RJ, Latinus M, Taylor MJ. Face, eye and object early processing: What is the face specificity? NeuroImage. 2006; 29:667-676.10.1016/j.neuroimage.2005.07.041 [PubMed: 16169749]

Itier RJ, Taylor MJ. Inversion and contrast polarity reversal affect both encoding and recognition processes of unfamiliar faces: A repetition study using ERPs. NeuroImage. 2002; 15:353372.10.1006/nimg.2001.0982 [PubMed: 11798271]

Itier RJ, Taylor MJ. N170 or N1? Spatiotemporal differences between object and face processing using ERPs. Cerebral Cortex. 2004; 14:132-142.10.1093/cercor/bhg111 [PubMed: 14704210] 
Itier RJ, Van Roon P, Alain C. Species sensitivity of early face and eye processing. NeuroImage. 2011; 54:705-713.10.1016/j.neuroimage.2010.07.031 [PubMed: 20650321]

Jemel B, George N, Chaby L, Fiori N, Renault B. Differential processing of part-to-whole and part-topart face priming: An ERP study. Neuroreport. 1999; 10:1069-1075. [PubMed: 10321486]

Kloth N, Schweinberger SR. Electrophysiological correlates of eye gaze adaptation. Journal of Vision. 2010; 10:1-13.10.1167/10.12.17

Kovács G, Zimmer M, Bankó É, Harza I, Antal A, Vidnyánszky Z. Electrophysiological correlates of visual adatation to faces and body parts in humans. Cerebral Cortex. 2006; 16:742-753.10.1093/ cercor/bhj020 [PubMed: 16120795]

Latinus M, Taylor MJ. Holistic processing of faces: Learning effects with Mooney faces. Journal of Cognitive Neuroscience. 2005; 17:1316-1327.10.1162/0898929055002490 [PubMed: 16197686]

McCarthy G, Puce A, Belger A, Allison T. Electrophysiological studies of human face perception. II: Response properties of face-specific potentials generated in occipitotemporal cortex. Cerebral Cortex. 1999; 9:431-444.10.1093/cercor/9.5.431 [PubMed: 10450889]

Op De Beeck H, Vogels R. Spatial sensitivity of macaque inferior temporal neurons. The Journal of Comparative Neurology. 2000; 426:505-518.10.1002/1096-9861 [PubMed: 11027395]

Perrett DI, Hietanen K, Oram W, Benson PJ, Rolls ET. Organization and functions of cells responsive to faces in the temporal cortex [and discussion]. Philosophical Transactions: Biological Sciences. 1992; 335:23-30.10.1098/rstb.1992.0003 [PubMed: 1348133]

Perrett DI, Oram W, Harries H, Bevan R, Hietanen K, Benson PJ, Thomas S. Viewer-centered and object-centered coding of heads in the macaque temporal cortex. Experimental Brain Research. 1991; 86:159-173.10.1007/BF00231050 [PubMed: 1756786]

Perrett DI, Rolls E, Caan W. Visual neurones to faces in the monkey temporal cortex. Experimental Brain Research. 1982; 47:329-342.10.1007/BF00239352 [PubMed: 7128705]

Perrett DI, Smith PAJ, Potter DD, Mistlin AJ, Head AS, Milner AD, Jeeves A. Visual cells in the temporal cortex sensitive to face view and gaze direction. Proceedings of the Royal Society of London. Series B, Biological Sciences. 1985; 223:293-317.10.1098/rspb.1985.0003

Puce A, Allison T, McCarthy G. Electrophysiological studies of human face perception. III: Effects of top-down processing on face-specific potentials. Cerebral Cortex. 1999; 9:445-458.10.1093/ cercor/9.5.445 [PubMed: 10450890]

Rhodes G, Jeffery L, Watson TL, Jaquet E, Winkler C, Clifford CWG. Orientation-contingent face aftereffects and implications for face-coding mechanisms. Current Biology. 2004; 14:2119_ 2123.10.1016/j.cub.2004.11.053 [PubMed: 15589154]

Rossion B, Campanella S, Gomez CM, Delinte A, Debatisse D, Liard L, Guerit JM. Task modulation of brain activity related to familiar and unfamiliar face processing: An ERP study. Clinical Neurophysiology. 1999; 110:449-462.10.1016/S1388-2457(98)00037-6 [PubMed: 10363769]

Rossion B, Delvenne F, Debatisse D, Goffaux V, Bruyer R, Crommelinck M, Guérit JM. Spatiotemporal localization of the face inversion effect: An event-related potentials study. Biological Psychology. 1999; 50:173-189.10.1016/S0301-0511(99)00013-7 [PubMed: 10461804]

Rossion B, Gauthier I. How does the brain process upright and inverted faces? Behavioral and Cognitive Neuroscience Reviews. 2002; 1:63-75.10.1177/1534582302001001004 [PubMed: 17715586]

Rossion B, Gauthier I, Tarr J, Despland P, Bruyer R, Linotte S, Crommelinck M. The N170 occipitotemporal component is delayed and enhanced to inverted faces but not to inverted objects: An electrophysiological account of face-specific processes in the human brain. Neuroreport. 2000; 11:69-72. [PubMed: 10683832]

Rossion B, Jacques C. Does physical interstimulus variance account for early electrophysiological face sensitive responses in the human brain? Ten lessons on the N170. NeuroImage. 2008; 39:19591979.10.1016/j.neuroimage.2007.10.011 [PubMed: 18055223]

Rousselet GA, Thorpe SJ, Fabre-Thorpe M. How parallel is visual processing in the ventral pathway? Trends in Cognitive Sciences. 2004; 8:363-370.10.1016/j.tics.2004.06.003 [PubMed: 15335463]

Sadeh B, Yovel G. Why is the N170 enhanced for inverted faces? An ERP competition experiment. NeuroImage. 2010; 53:782-789.10.1016/j.neuroimage.2010.06.029 [PubMed: 20558303] 
Sagiv N, Bentin S. Structural encoding of human and schematic faces: Holistic and part-based processes. Journal of Cognitive Neuroscience. 2001; 13:937-951.10.1162/089892901753165854 [PubMed: 11595097]

Schyns PG, Petro LS, Smith L. Dynamics of visual information integration in the brain for categorizing facial expressions. Current Biology. 2007; 17:1580-1585.10.1016/j.cub.2007.08.048 [PubMed: 17869111]

Schyns PG, Petro LS, Smith L. Transmission of facial expressions of emotion co-evolved with their efficient decoding in the brain: Behavioral and brain evidence. PLoS One. 2009; 4:1-16.10.1371/ journal.pone.0005625

Sekuler AB, Gaspar CM, Gold M, Bennett P. Inversion leads to quantitative, not qualitative, changes in face processing. Current Biology. 2004; 14:391-396.10.1016/j.cub.2004.02.028 [PubMed: 15028214]

Taylor J, Itier RJ, Allison T, Edmonds GE. Direction of gaze effects on early face processing: Eyesonly versus full faces. Cognitive Brain Research. 2001; 10:333-340.10.1016/ S0926-6410(00)00051-3 [PubMed: 11167057]

Tsao DY, Livingstone MS. Mechanisms of face perception. Annual Review of Neuroscience. 2008; 31:411-437.10.1146/annurev.neuro.30.051606.094238

Watanabe S, Kakigi R, Puce A. The spatiotemporal dynamics of the face inversion effect: A magnetoand electro-encephalographic study. Neuroscience. 2003; 116:879-895.10.1016/ S0306-4522(02)00752-2 [PubMed: 12573727]

Willenbockel V, Sadr J, Fiset D, Horne G, Gosselin F, Tanaka JW. Controlling low-level image properties: The SHINE toolbox. Behavior Research Methods. 2010; 42:671-684.10.3758/BRM. 42.3.671 [PubMed: 20805589]

Williams CC, Henderson JM. The face inversion effect is not a consequence of aberrant eye movements. Memory \& Cognition. 2007; 35:1977-1985.10.3758/BF03192930 [PubMed: 18265613]

Yin RK. Looking at upside-down faces. Journal of Experimental Psychology. 1969; 81:141145.10.1037/h0027474

Yovel G, Kanwisher N. The neural basis of the behavioral face-inversion effect. Current Biology. 2005; 15:2256-2262.10.1016/j.cub.2005.10.072 [PubMed: 16360687] 


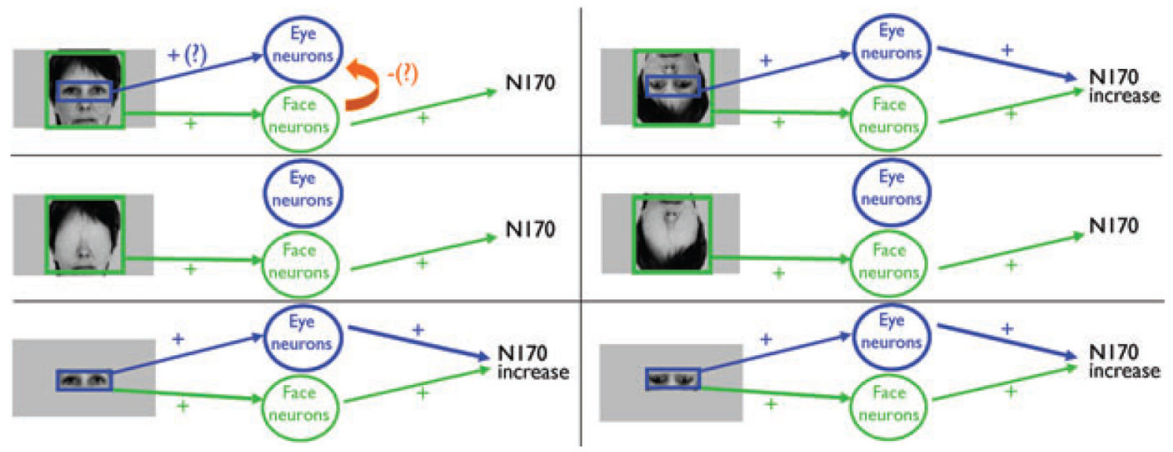

Figure 1.

Simplified neural model of N170 amplitude modulations during early face processing stages adapted from Itier and Batty (2009). N170 is assumed to arise from the activation of facesensitive and eye-sensitive neurons likely coexisting in the Fusiform Gyrus and Superior Temporal Sulcus regions of the human brain. The ' + ' signs signify neuronal activation. Both face and eye-sensitive neurons respond to eyes in isolation but only face-sensitive neurons respond to full faces. The '-' sign and question mark refer to a possible inhibition mechanism exacted by face-sensitive neurons onto eye-sensitive neurons, preventing them from responding to the eyes presented within an upright face configuration. The disruption of facial configuration in inverted faces allows eye-sensitive neurons to respond to the eyes, triggering an increase in N170 amplitude. For a full description of the model, see Itier $\mathrm{et} \mathrm{al.}$ (2007) and Itier and Batty (2009). 


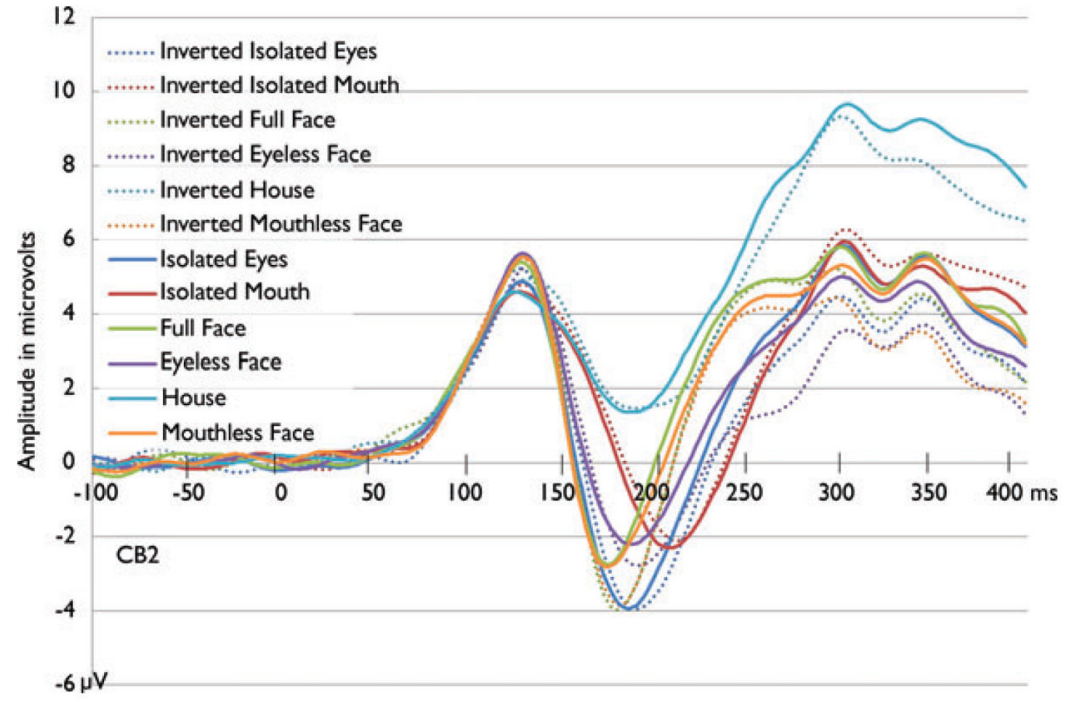

Figure 2.

Grand-averaged ERP obtained after S1 (adaptor) onset at right posterior electrode CB2, shown for all the categories in upright (solid) and inverted (dotted) orientation. 


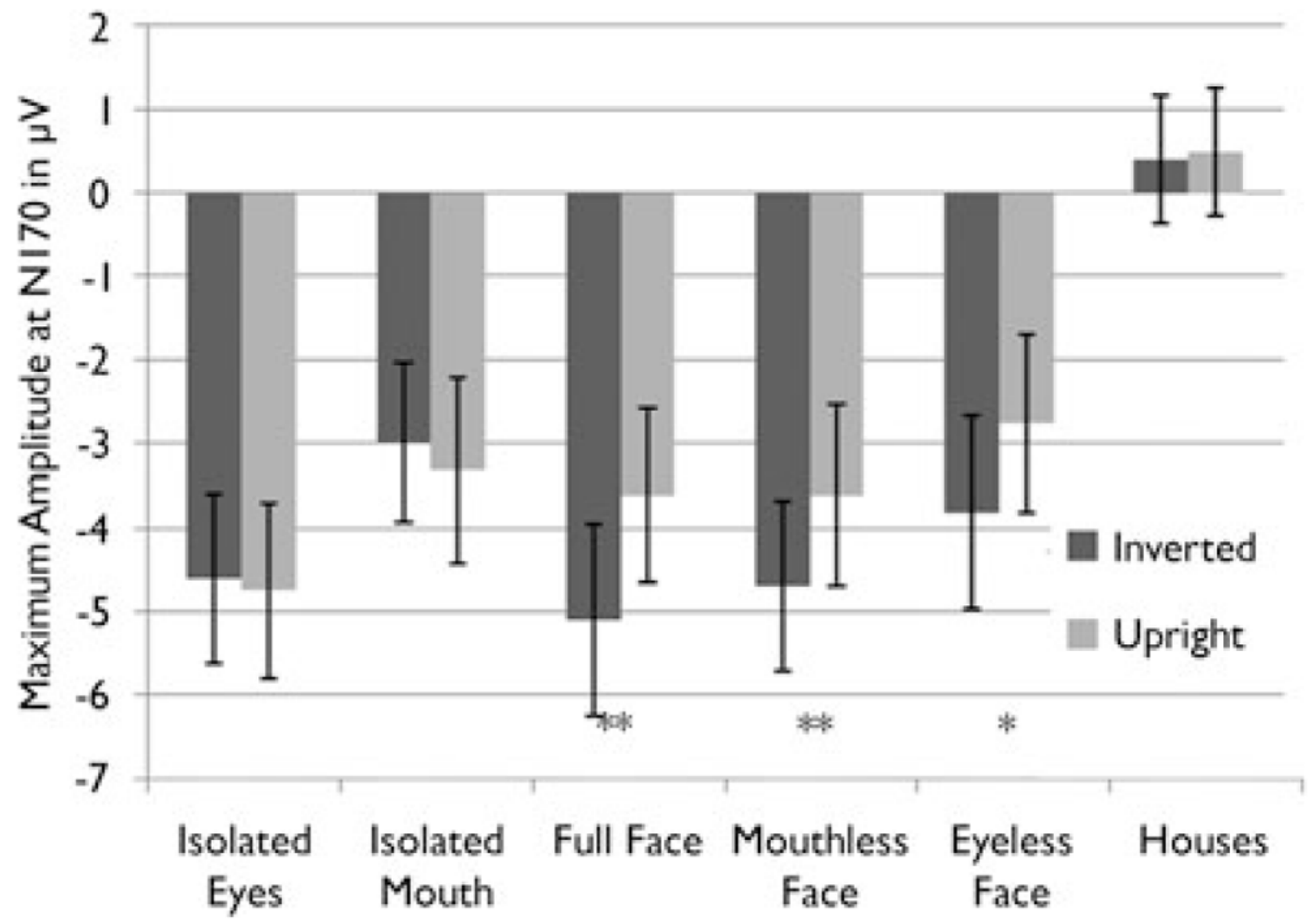

Figure 3.

Grand-averaged maximum amplitude of N170 peak following S1 onset (adaptor stimulus) at posterior electrodes CB1 and CB2 combined. ${ }^{*} p<.05 ;{ }^{* *} p<.01$. 


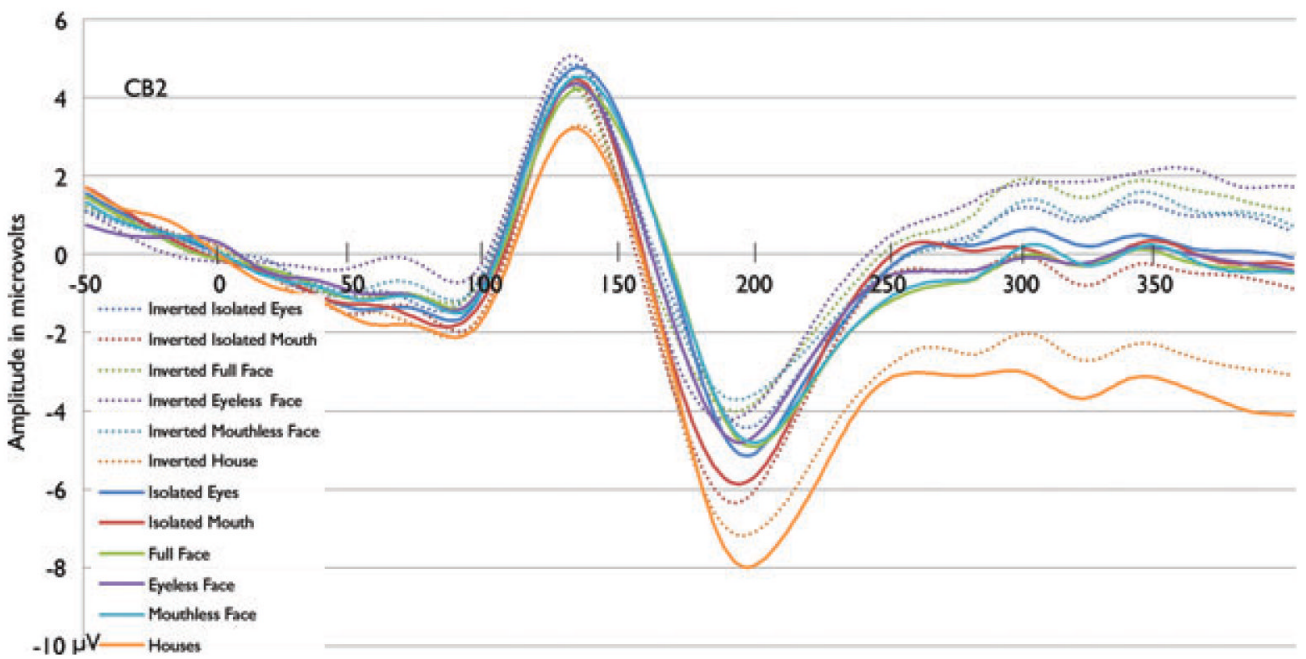

Figure 4.

Grand-averaged ERP obtained after S2 (inverted face test stimulus) onset at right posterior electrode CB2, shown for all the categories of preceding adaptors in upright (solid) and inverted (dotted) presentation. 


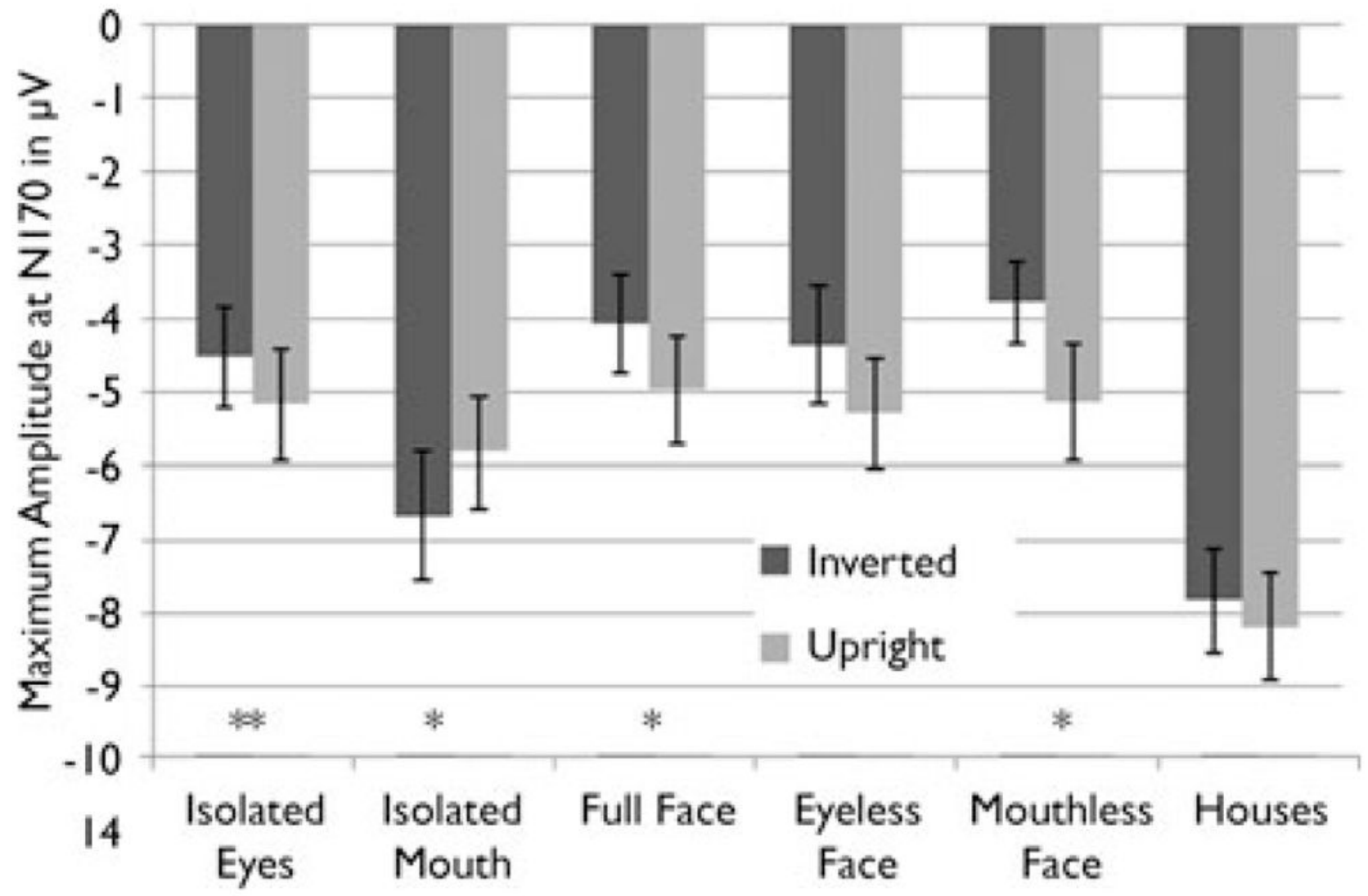

Figure 5.

Grand-averaged maximum amplitude of N170 peak following S2 (test stimulus) onset at posterior electrodes CB1 and CB2 combined. ${ }^{*} p<.05 ;{ }^{* *} p<.01$. 